\title{
A quantum topological phase transition at the microscopic level
}

\author{
Claudio Castelnovo ${ }^{1}$ and Claudio Chamon ${ }^{2}$ \\ ${ }^{1}$ Rudolf Peierls Centre for Theoretical Physics, University of Oxford, Oxford, OX1 3NP, UK \\ 2 Physics Department, Boston University, Boston, MA 02215, USA
}

(Dated: October 23, 2018)

\begin{abstract}
We study a quantum phase transition between a phase which is topologically ordered and one which is not. We focus on a spin model, an extension of the toric code, for which we obtain the exact ground state for all values of the coupling constant that takes the system across the phase transition. We compute the entanglement and the topological entropy of the system as a function of this coupling constant, and show that the topological entropy remains constant all the way up to the critical point, and jumps to zero beyond it. Despite the jump in the topological entropy, the transition is second order as detected via local observables.
\end{abstract}

\section{INTRODUCTION}

Some strongly correlated quantum many body systems display a type of order which cannot be characterized by any local order parameter. Instead, such order is topological in nature ${ }^{1}$, with the fractional quantum Hall systems being the primary example so far: they are liquid states that exhibit exotic properties such as a ground state (GS) degeneracy that cannot be lifted by any local perturbations ${ }^{2,3}$ and fractionalized degrees of freedom ${ }^{4}$. Topologically ordered states are also interesting in that their robustness against local perturbations might be of use for decoherence-free quantum computation ${ }^{5}$.

An example of an exactly solvable lattice spin model that is topologically ordered was presented by Kitaev in Ref. 5, and the system was argued to be robust against small perturbations that tend to order the system à la Landau-Ginzburg and take it away from its topological phase. The departure from the topologically ordered phase should occur through a quantum phase transition. Such quantum phase transition, however, cannot be entirely captured by ordinary methods based on local Landau-Ginzburg order parameters, and new methods need to be devised in order to investigate the fate of topological order across the phase transition. These novel methods must be based on the fundamental properties of topologically ordered phases, such as the GS degeneracy in presence of a gap, and the presence of a non-vanishing topological entropy.

Recent efforts to understand quantum phase transitions in topologically ordered states include a mean-field approach for these exotic states 6 , and analytical and numerical studies ${ }^{7,8,9}$ of the Kitaev model in the presence of a field. The numerical analysis presented in Refs. 8.9 leads to the conclusion that topological order survives unchanged up to the second order phase transition at $\beta_{c}=0.32847(6)$ (in the notation of Eq. (18)), while the system is no longer topologically ordered for $\beta>\beta_{c}$. (Here $\beta$ stands for the coupling constant that drives the $T=0$ quantum phase transition - the notation will become apparent shortly, and is chosen because of a close relation to a classical model.)

In this paper, we investigate analytically a quantum phase transition out of a topological phase. We show that the recently defined topological entropy ${ }^{10,11}$ works well as an "order parameter" across the transition. We study the transition using a model - see Eq. (5) - that is shown to behave much like the Kitaev model in a magnetic field for small values of the field.
The advantage of this model is that the ground state can be obtained exactly, from which we can then compute the topological entropy explicitly, and show that it remains constant in the topologically ordered phase $\left(\beta<\beta_{c} \simeq 0.4406868\right.$ ), dropping abruptly to zero in the non-topologically-ordered phase $\left(\beta>\beta_{c}\right)$, despite the continuous (second order) character of the transition.

We find that in this model, even though one cannot identify a local order parameter that vanishes in one phase and not in the other, one can show that the (continuous) local magnetization has a singularity at the critical point. In the model, we show that the magnetization equals the energy $E_{\mathrm{Ising}}(\beta)$ of a $2 D$ classical Ising model with $N$ spins evaluated at an inverse (classical) temperature equal to the value of the coupling constant $\beta$ that drives the system through the $T=0$ phase transition:

$$
m(\beta)=\frac{1}{N} \sum_{i}\left\langle\hat{\sigma}_{i}^{\mathrm{z}}\right\rangle=\frac{1}{N} E_{\mathrm{Ising}}(\beta) .
$$

From this relation, it becomes evident that the magnetization $m(\beta)$, although continuous and non-vanishing across the transition at $\beta_{c}$ (much as the energy of the classical Ising model across the classical transition), has a singularity in its first derivative, since

$$
\frac{\partial m}{\partial \beta}=\frac{1}{N} \frac{\partial E_{\mathrm{Ising}}}{\partial \beta}=-\beta^{2} \frac{1}{N} C_{\mathrm{Ising}}(\beta),
$$

and the Ising model heat capacity $C_{\text {Ising }}$ diverges logarithmically at $\beta_{c}$. Hence, although there is no local order parameter that can detect either the topological or the non-topological phase in this system, one can expose the topological quantum phase transition through the singularity in the derivative of a local quantity.

This is contrasted, for example, with the case discussed in $\mathrm{Sec}$ IIIB, where a similar topological transition is accompanied by a simultaneous $\mathbb{Z}_{2}$ symmetry breaking phase transition. In that case, the same transition is captured both by the non-local topological entropy, and by a local (LandauGinzburg) order parameter.

\section{THE MODEL}

The model that we consider is a deformation of the Kitaev model $^{5}$, and it is defined on a square lattice with spin- $1 / 2$ de- 
grees of freedom living on the bonds, as shown in Fig. 1 The pure Kitaev model is written in terms of star and plaquette operators (see Fig. 1). Star operators are defined as

$$
A_{s}=\prod_{i \in \operatorname{star}(s)} \hat{\sigma}_{i}^{\mathrm{x}} \equiv \prod_{i \in s} \hat{\sigma}_{i}^{\mathrm{x}}
$$

where $i$ labels the four spins on the bonds departing from some vertex $s$ of the square lattice. Plaquette operators are defined as

$$
B_{p}=\prod_{i \in \text { plaquette }(p)} \hat{\sigma}_{i}^{\mathrm{z}} \equiv \prod_{i \in p} \hat{\sigma}_{i}^{\mathrm{z}}
$$

where $i$ labels the four spins on the bonds around some plaquette $p$ of the square lattice.

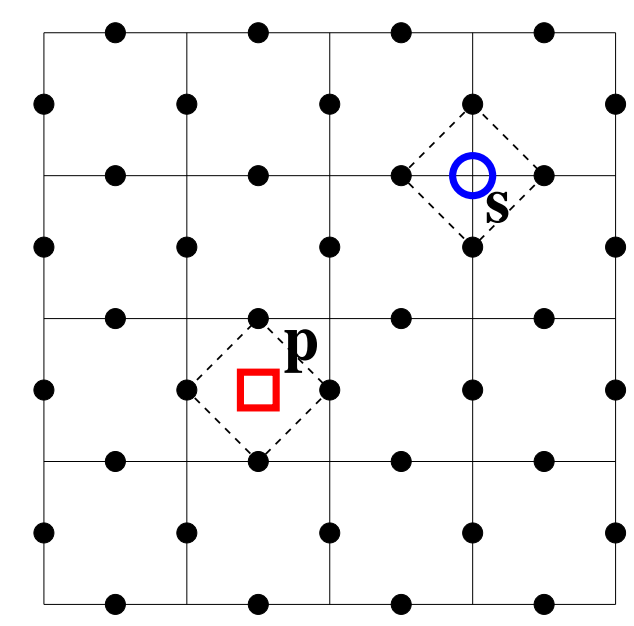

FIG. 1: (Color online) - Examples of star and plaquette operators, centered at a lattice site $s$ (blue open circle) and at a dual lattice site $p$ (red open square), respectively. The solid black dots represent the spin- $1 / 2$ degrees of freedom living on the bonds of the lattice, and the dashed lines connect the spins involved in the definition of each of the above operators.

The Hamiltonian we consider in this paper is

$$
\begin{aligned}
H & =-\lambda_{0} \sum_{p} B_{p}-\lambda_{1} \sum_{s} A_{s}+\lambda_{1} \sum_{s} e^{-\beta \sum_{i \in s} \hat{\sigma}_{i}^{z}} \\
& =H_{\text {Kitaev }}+\lambda_{1} \sum_{s} e^{-\beta \sum_{i \in s} \hat{\sigma}_{i}^{z}}
\end{aligned}
$$

where $\lambda_{0,1}>0$ and $\beta$ is a parameter that we use to tune the system across a topological quantum phase transition. Notice that for $\beta=0$ the Hamiltonian Eq. (5) is simply the Kitaev Hamiltonian $H_{\text {Kitaev }}$ in $\operatorname{Ref} 5$, up to a trivial overall constant shift of the energy.

The exact ground state wavefunction of this Hamiltonian can be obtained by deconstructing $H$ into two pieces, $H=$ $\lambda_{0} H_{0}+\lambda_{1} H_{1}$, as follows.

Take $G$ to be the (Abelian) group of all spin flip operations obtained as products of star type operators. Notice that $g^{2}=1$ for any element $g$ of the group $G$. By acting with elements of $G$ on a given reference configuration $\bigotimes_{i}\left|\sigma_{i}^{z}\right\rangle$ one generates a manifold of states, which however does not encompass the whole basis. For example, the action of a star operator $A_{s}$ cannot change the sign of the product of $\sigma^{\mathrm{z}}$ 's around any square plaquette in the lattice ${ }^{5.12}$ (see Fig. 11). Therefore, there is a non-trivial (and non-unique) minimal set $\left\{\left|\Psi_{\alpha}\right\rangle\right\}$ of reference configurations that generates the full $\sigma^{\mathrm{z}}$-basis under the action of the group $G$. (In particular, one of the elements in this set is the reference configuration $|0\rangle$ that is fully magnetized in the $z$-direction, say $\sigma_{i}^{z}=1, \forall i$.)

Consider then the family of Hamiltonians

$$
H_{1}(\beta)=\sum_{s}\left[e^{-\beta \sum_{i \in s} \hat{\sigma}_{i}^{z}}-\prod_{i \in s} \hat{\sigma}_{i}^{\mathrm{x}}\right],
$$

for some real-valued parameter $\beta$. The ground state of any such Hamiltonian can be obtained exactly and it can be written in the form

$$
\begin{aligned}
\left|G S_{1}\right\rangle & =\sum_{\alpha} \psi_{\alpha} \sum_{g \in G} \frac{e^{\beta \sum_{i} \sigma_{i}^{z}(g, \alpha) / 2}}{\sqrt{Z_{\alpha}}} g\left|\Psi_{\alpha}\right\rangle, \\
Z_{\alpha} & =\sum_{g \in G} e^{\beta \sum_{i} \sigma_{i}^{z}(g, \alpha)}
\end{aligned}
$$

where $\alpha$ labels the different block-diagonal sectors corresponding to the states in the minimal set $\left\{\left|\Psi_{\alpha}\right\rangle\right\} ; \sigma_{i}^{z}(g, \alpha)$ is the $z$-component of the spin at site $i$ in state $g\left|\Psi_{\alpha}\right\rangle$; and the coefficients $\psi_{\alpha}$ can be chosen at will, subject to the normalization condition $\sum_{\alpha}\left|\psi_{\alpha}\right|^{2}=1$. Although the choice of minimal set is non-unique, one can show that Eq. (8) is independent of such choice, modulo an irrelevant permutation of the $\alpha$ indices. Within each block-diagonal sector, the GS of Eq. (6) is unique. Instead of proving directly that (8) is the GS of (6), it is more convenient to notice that the family of Hamiltonians in Eq. (6) is a particular choice of Stochastic Matrix Form decompositions of quantum Hamiltonians that exhibit precisely Eq. (8) as their GS $\underline{\underline{13}}$ One can verify this by showing that each of the operators

$$
Q_{s}=e^{-\beta \sum_{i \in s} \hat{\sigma}_{i}^{2}}-\prod_{i \in s} \hat{\sigma}_{i}^{\mathrm{x}}
$$

between square brackets in Eq. (6) annihilates the inner sum in $\left|G S_{1}\right\rangle$, independently of the index $\alpha$. That the GS energy is zero follows because

$$
Q_{s}{ }^{2}=2 \cosh \left(\beta \sum_{i \in s} \hat{\sigma}_{i}^{z}\right) Q_{s}
$$

and

$$
\left[Q_{s}, \cosh \left(\beta \sum_{i \in s} \hat{\sigma}_{i}^{\mathrm{z}}\right)\right]=0,
$$

from which it can be shown that the expectation value of $Q_{s}$ with respect to any state is always greater than or equal to zero. 
Let us consider now the remaining part of the Hamiltonian,

$$
H_{0}=-\sum_{p} \prod_{i \in \text { plaquette }(p)} \hat{\sigma}_{i}^{\mathrm{z}} \equiv-\sum_{p} \prod_{i \in p} \hat{\sigma}_{i}^{\mathrm{z}} .
$$

Recall that any star operator $A_{s}$, and therefore any element of the group $G$, preserves the product $\prod_{i \in p} \hat{\sigma}_{i}^{z}$ on every plaquette of the lattice. The GS wavefunction of Hamiltonian (12) can then be written as

$$
\left|G S_{0}\right\rangle=\sum_{\alpha}^{\prime} \sum_{g \in G} \phi_{g, \alpha} g\left|\Psi_{\alpha}\right\rangle,
$$

for any choice of the coefficients $\phi_{g, \alpha}\left(\sum_{\alpha}{ }^{\prime} \sum_{g \in G}\left|\phi_{g, \alpha}\right|^{2}=\right.$ 1 ). Here the primed sum over $\alpha$ is restricted to the (four) block-diagonal sectors that satisfy $\prod_{i \in p} \hat{\sigma}_{i}^{z}=+1$ for all plaquettes $p$ in the lattice, and it must be carried out separately because no operation in $G$ allows to change sector.

As a result, any linear combination with positive weights $\lambda_{0}$ and $\lambda_{1}$,

$$
\begin{aligned}
H & =\lambda_{0} H_{0}+\lambda_{1} H_{1} \\
& =H_{\text {Kitaev }}+\lambda_{1} \sum_{s} e^{-\beta \sum_{i \in s} \hat{\sigma}_{i}^{z}},
\end{aligned}
$$

and therefore our Hamiltonian in Eq. (5), has the GS given by

$$
|G S\rangle=\sum_{\alpha}^{\prime} \psi_{\alpha} \sum_{g \in G} \frac{e^{\beta \sum_{i} \sigma_{i}^{z}(g, \alpha) / 2}}{\sqrt{Z_{\alpha}}} g\left|\Psi_{\alpha}\right\rangle .
$$

Notice that one of the topological sectors that satisfy $\prod_{i \in p} \hat{\sigma}_{i}^{z}=+1, \forall p$ is the one containing the fully magnetized configuration in the $z$-direction $(|0\rangle)$.

In particular for $|\beta| \ll 1$

$$
\begin{aligned}
e^{-\beta \sum_{i \in s} \hat{\sigma}_{i}^{\mathrm{z}}} & \simeq 1-\beta \sum_{i \in s} \hat{\sigma}_{i}^{\mathrm{z}} \\
\lambda_{1} \sum_{s} e^{-\beta \sum_{i \in s} \hat{\sigma}_{i}^{\mathrm{z}}} & \simeq \mathrm{const}-2 \beta \lambda_{1} \sum_{i} \hat{\sigma}_{i}^{\mathrm{z}} .
\end{aligned}
$$

Therefore, in the limit of small $\beta$ (in absolute value) the Hamiltonian in Eqs. (5]14) is equivalent to the Kitaev model in presence of a magnetic field proportional to $\beta \lambda_{1}$,

$$
H=-\lambda_{0} \sum_{p} \prod_{i \in p} \hat{\sigma}_{i}^{\mathrm{z}}-\lambda_{1} \sum_{s} \prod_{i \in s} \hat{\sigma}_{i}^{\mathrm{x}}-2 \beta \lambda_{1} \sum_{i} \hat{\sigma}_{i}^{\mathrm{Z}} .
$$

For larger values of $\beta$, the many-body terms in Eq. (19) are no longer negligible and the equivalence is lost, although the form of the GS (15) suggests that the system gets deeper and deeper into the magnetized phase - as one would expect upon increasing the strength of the magnetic field in the Kitaev model. As we discuss in Section IIIA our model undergoes a second-order phase transition at $\beta_{c}=(1 / 2) \ln (\sqrt{2}+1) \simeq$ 0.4406868 , where it displays a dimensionality reduction that places the transition in a different universality class than the one studied in Refs. $7,8,9$.
One can use the decomposition

$$
\begin{aligned}
e^{-\beta \sum_{i \in s} \hat{\sigma}_{i}^{z}} & =\prod_{i \in s}\left[\cosh (\beta)-\hat{\sigma}_{i}^{\mathrm{z}} \sinh (\beta)\right] \\
& =\cosh ^{4}(\beta) \\
& -\cosh ^{3}(\beta) \sinh (\beta) \sum_{i \in s} \hat{\sigma}_{i}^{\mathrm{z}} \\
& +\cosh ^{2}(\beta) \sinh ^{2}(\beta) \sum_{i \neq j \in s} \hat{\sigma}_{i}^{\mathrm{z}} \hat{\sigma}_{j}^{\mathrm{z}} \\
& -\cosh (\beta) \sinh ^{3}(\beta) \sum_{i \neq j \neq k \in s} \hat{\sigma}_{i}^{\mathrm{z}} \hat{\sigma}_{j}^{\mathrm{z}} \hat{\sigma}_{k}^{\mathrm{z}} \\
& +\sinh ^{4}(\beta) \prod_{i \in s} \hat{\sigma}_{i}^{\mathrm{z}},
\end{aligned}
$$

to estimate the limit of validity of Eq. (18) to be given by the condition

$$
\left|\frac{\cosh ^{3}(\beta) \sinh (\beta)}{\cosh ^{2}(\beta) \sinh ^{2}(\beta)}\right|=\left|\frac{\cosh (\beta)}{\sinh (\beta)}\right| \gtrsim 2 .
$$

This corresponds to a ratio between the coupling to the magnetic field and the coupling to the cooperative transverse field $\left(\sum_{s} \prod_{i \in s} \hat{\sigma}_{i}^{\mathrm{x}}\right)$

$$
\left|\frac{2 \lambda_{1} \cosh ^{3}(\beta) \sinh (\beta)}{\lambda_{1}}\right| \lesssim \frac{16}{9} \simeq 1.78 .
$$

The detailed numerical analysis presented in Refs. 8.9 lead the authors to conclude that topological order survives up to the second order phase transition at finite $\beta_{c}$ (in the notation of Eq. (18)), while the system is no longer topologically ordered for $\beta>\beta_{c}$. In the following, we investigate this phase and the relative phase transition using the exact ground state of our model (5) to compute the topological entropy ${ }^{10,11}$ across the transition. Using an exact derivation from the microscopic degrees of freedom, we show that the topological entropy is able to detect a transition from a topologically ordered phase $\left(\beta<\beta_{c}\right)$ to a non-topologically-ordered phase $\left(\beta>\beta_{c}\right)$. Indeed, it remains constant at its known $\beta \rightarrow 0$ value up to the transition and drops abruptly to zero afterwards, despite the continuous character of the transition.

\section{THE TOPOLOGICAL ENTROPY OF FACTORIZABLE (LOCAL) WAVEFUNCTIONS}

Using the definition in Refs. 10.11, the topological entropy can be obtained as a linear combination of Von Neumann entanglement entropies $S_{\mathrm{VN}}$ of different bipartitions of the system into subsystems $A$ and $B$ :

$$
S_{\mathrm{VN}}^{A} \equiv-\operatorname{Tr}\left[\rho_{A} \log _{2} \rho_{A}\right]=S_{\mathrm{VN}}^{B},
$$

where $\rho_{A}=\operatorname{Tr}_{B}(\rho)$ is the reduced density matrix obtained from the full density matrix $\rho$ by tracing out the degrees of freedom of subsystem $B$, and the last equality holds whenever the full density matrix $\rho$ is a pure-state density matrix. 
The different bipartitions are aimed at removing all the extensive (boundary) contributions to uncover the sole topological contribution. A particular choice of the four bipartitions ${ }^{10}$ is illustrated in Fig. 2, and the topological entropy is then defined

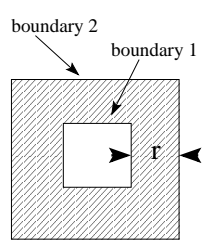

(1)

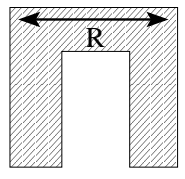

(2)

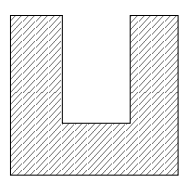

(3)

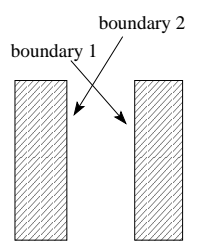

(4)
FIG. 2: Illustration of the four bipartitions used to compute the topological entropy in Ref. 10 .

as:

$$
S_{\mathrm{topo}}=\lim _{r, R \rightarrow \infty}\left(-S_{\mathrm{VN}}^{(1 A)}+S_{\mathrm{VN}}^{(2 A)}+S_{\mathrm{VN}}^{(3 A)}-S_{\mathrm{VN}}^{(4 A)}\right) .
$$

In order to compute the topological entropy as a function of the parameter $\beta$, let us first notice that the Hamiltonian (14) constructed above, with GS given by (15), belongs to a class of Hamiltonians whose GS wavefunctions $|\Psi\rangle=|Z|^{-1 / 2} \sum_{g \in G} e^{-\beta E_{g} / 2} g|0\rangle$ have non-negative, factorizable amplitudes, i.e., $E_{g}=E_{g_{A}}^{A}+E_{g_{B}}^{B}$, with $g=g_{A} \otimes g_{B}$ for all bipartitions $(A, B)$. For this type of Hamiltonians, one can compute the entanglement entropy as follows.

Consider a given bipartition $(A, B)$ of the system. The reduced density matrix $\rho_{A}=\operatorname{Tr}_{B}(\rho)$, obtained by tracing over all degrees of freedom in $B$, is given by ${ }^{12}$

$$
\begin{aligned}
\rho_{A} & =\frac{1}{Z} \sum_{g, \tilde{g} \in G} e^{-\beta\left(E_{g}+E_{g \tilde{g}}\right) / 2} \\
& \times\left\langle 0_{B}\left|g_{B} \tilde{g}_{B} g_{B}\right| 0_{B}\right\rangle g_{A}\left|0_{A}\right\rangle\left\langle 0_{A}\right| g_{A} \tilde{g}_{A} \\
& =\frac{1}{Z} \sum_{g \in G, g^{\prime} \in G_{A}} e^{-\beta E_{g_{B}}^{B}} e^{-\beta\left(E_{g_{A}}^{A}+E_{g_{A} g_{A}^{\prime}}^{A}\right) / 2} \\
& \times g_{A}\left|0_{A}\right\rangle\left\langle 0_{A}\right| g_{A} g_{A}^{\prime} .
\end{aligned}
$$

where $g=g_{A} \otimes g_{B},|0\rangle=\left|0_{A}\right\rangle \otimes\left|0_{B}\right\rangle$ and $G_{A} \subset G\left(G_{B} \subset\right.$ $G)$ is the subgroup of transformations acting solely on $A(B)$ and leaving $B(A)$ invariant:

$$
\begin{aligned}
& G_{A}=\left\{g \in G \mid g_{B}=\mathbf{1}_{B}\right\} \\
& G_{B}=\left\{g \in G \mid g_{A}=\mathbf{1}_{A}\right\}
\end{aligned}
$$

Notice that we used the group property to rewrite a generic element of $G$ as $g \tilde{g}, \exists ! \tilde{g} \in G$, as well as the additive property of $E_{g}$.

We can then compute the trace of the $n$-th power of the reduced density matrix $\operatorname{Tr}\left[\left(\rho_{A}\right)^{n}\right]$ and use the identity

$$
-\lim _{n \rightarrow 1} \frac{\partial}{\partial n} \operatorname{Tr}\left[\left(\rho_{A}\right)^{n}\right]=-\operatorname{Tr}\left[\rho_{A} \ln \rho_{A}\right]
$$

to obtain the Von Neumann entropy $S_{\mathrm{VN}}^{(A)}=-\operatorname{Tr}\left[\rho_{A} \log _{2} \rho_{A}\right]$ :

$$
\begin{aligned}
\operatorname{Tr}\left[\rho_{A}^{n}\right]= & \frac{1}{Z^{n}} \sum_{\substack{g_{1}, \ldots, g_{n} \in G \\
g_{1}^{\prime}, \ldots, g_{n}^{\prime} \in G_{A}}} e^{-\beta \sum_{i=1}^{n} E_{g_{i, B}}^{B}} \\
& \times e^{-\beta \sum_{i=1}^{n}\left(E_{g_{i, A}}^{A}+E_{g_{i, A} g_{i, A}^{\prime}}^{A}\right) / 2} \\
& \times\left\langle 0_{A}\left|g_{1, A} g_{1, A}^{\prime} g_{2, A}\right| 0_{A}\right\rangle\left\langle 0_{A}\left|g_{2, A} g_{2, A}^{\prime} g_{3, A}\right| 0_{A}\right\rangle \ldots \\
& \times \ldots\left\langle 0_{A}\left|g_{n, A} g_{n, A}^{\prime} g_{1, A}\right| 0_{A}\right\rangle \\
= & \frac{1}{Z^{n}} \sum_{g_{1}, \ldots, g_{n} \in G} e^{-\beta \sum_{i=1}^{n} E_{g_{i}}} \\
& \times\left\langle 0_{A}\left|g_{1, A} g_{1, A}^{\prime} g_{2, A}\right| 0_{A}\right\rangle\left\langle 0_{A}\left|g_{2, A} g_{2, A}^{\prime} g_{3, A}\right| 0_{A}\right\rangle \ldots \\
& \times \ldots\left\langle 0_{A}\left|g_{n, A} g_{n, A}^{\prime} g_{1, A}\right| 0_{A}\right\rangle,
\end{aligned}
$$

where we used the fact that the inner products in Eq. 26 impose

$$
g_{i+1, A}=g_{i, A} g_{i, A}^{\prime},
$$

for $i=1, \ldots, n$, with the identification $n+1 \equiv 1$, and therefore

$$
E_{g_{i, B}}^{B}+\frac{1}{2}\left(E_{g_{i, A}}^{A}+E_{g_{i-1, A} g_{i-1, A}^{\prime}}^{A}\right)=E_{g_{i}} .
$$

The condition in Eq. (27) can be satisfied if and only if

$$
g_{i}^{\prime}=\left(g_{i+1, A} g_{i, A}\right) \otimes \mathbf{1}_{B} \in G_{A} .
$$

Thus, the summation over all $g_{i}^{\prime}$ of the inner products in Eq. 26 yields a constraint over the allowed values of $g_{1}, \ldots, g_{n} \in G$ :

$$
\begin{gathered}
\left(g_{i+1, A} g_{i, A}\right) \otimes \mathbf{1}_{B} \in G_{A}, \quad \forall i=1, \ldots, n \quad(n+1 \equiv 1) \\
\left(g_{i, A} g_{j, A}\right) \otimes \mathbf{1}_{B} \in G_{A}, \quad \forall i, j=1, \ldots, n \\
g_{i} g_{j} \in G_{A} G_{B}, \stackrel{\mathbb{\Downarrow}}{\forall} \quad \forall i, j=1, \ldots, n .
\end{gathered}
$$

In particular, the last line can be recast as

$$
g_{i}=h_{i} g_{1} k_{i} \quad \exists ! h_{i} \in G_{A}, k_{i} \in G_{B}, \quad \forall i=2, \ldots, n .
$$

The physical meaning of these conditions will become clear in the next section, for the specific case of the system considered in this paper, although the form of Eq. (31) already suggests that they require all the elements $g_{i}$ to agree at the boundary of the bipartition $(A, B)$.

We can finally use Eq. (31) to simplify Eq. (26)

$$
\begin{aligned}
\operatorname{Tr}\left[\rho_{A}^{n}\right] & =\frac{1}{Z^{n}} \sum_{g \in G} e^{-\beta E_{g}} \sum_{\substack{h_{2}, \ldots, h_{n} \in G_{A} \\
k_{2}, \ldots, k_{n} \in G_{B}}} e^{-\beta \sum_{i=2}^{n} E_{h_{i} g k_{i}}} \\
& =\frac{1}{Z} \sum_{g \in G} e^{-\beta E_{g}}\left(\frac{\sum_{h \in G_{A}, k \in G_{B}} e^{-\beta E_{h g k}}}{Z}\right)^{n-1}
\end{aligned}
$$


and obtain, via Eq. 25),

$$
\begin{aligned}
& S_{\mathrm{VN}}^{(A)}=-\frac{1}{Z} \sum_{g \in G} e^{-\beta E_{g}} \log _{2}\left[\frac{\sum_{h \in G_{A}}, k \in G_{B} e^{-\beta E_{h g k}}}{Z}\right] \\
& =\left\langle\log _{2}\left[\frac{\sum_{h \in G_{A}}, k \in G_{B} e^{-\beta E_{h g k}}}{Z}\right]\right\rangle \\
& =-\left\langle\log _{2} \tilde{Z}_{g}\right\rangle+\log _{2} Z=\beta\left(\left\langle\tilde{F}_{g}\right\rangle-F\right) \text {, }
\end{aligned}
$$

where $\tilde{F}_{g}$ is the partial free energy given by all the configurations that can be obtained from $g$ via products of spin flip operators that act solely on subsystem $A$ or subsystem $B$ (i.e., having the same 'boundary' as $g$ ), and $\langle\ldots\rangle$ denotes the ensemble average over $g \in G$ with weight $e^{-\beta E_{g}}$. Notice that our result in Eq. (33) is the lattice equivalent of the Von Neumann entropy obtained by Fradkin and Moore in Ref. 14 for continuous systems.

Alternatively, Eq. (33) can be interpreted as the entropy of mixing (or configurational entropy) of the allowed bipartition boundaries in $G$. This can be made more transparent by introducing the quotient group $Q=G /\left(G_{A} G_{B}\right)$, and by rewriting Eq. (33) as

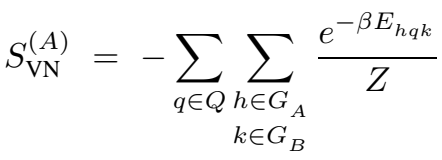

$$
\begin{aligned}
& \times \log _{2}\left[\frac{\sum_{\tilde{h} \in G_{A}}, \tilde{k} \in G_{B} e^{-\beta E_{\tilde{h}(h q k) \tilde{k}}}}{Z}\right] \\
& =-\sum_{q \in Q} P_{q} \log _{2} P_{q},
\end{aligned}
$$

where we used the fact that the term in square brackets is independent of $h$ and $k$, and where we introduced the notation

$$
P_{q}=\frac{\sum_{h \in G_{A}, k \in G_{B}} e^{-\beta E_{h q k}}}{Z}
$$

for the probability of boundary $q$ to appear in $G$, for a given inverse temperature $\beta$ and energy $E_{g}$.

In order to proceed further, let us focus for simplicity on the specific GS of our system (15). The generic case of a wavefunction with factorizable amplitudes can be inferred with minor modifications.

\section{A. The case of 1-body potentials}

All of the above results apply straightforwardly to the GS in Eq. (15). Notice that (i) the topological entropy in each block-diagonal sector of the pure Kitaev model is the same,,$\frac{12}{,}$ and (ii) it is reasonable to make the working assumption that the relevant sector for the transition to the fully magnetized state $|0\rangle$ is the one that contains this state, and that is therefore obtained upon applying the group $G$ to $|0\rangle$. For the purpose of computing the topological entropy, one can thus replace Eq. (15) by

$$
|G S\rangle=\frac{1}{\sqrt{Z}} \sum_{g \in G} e^{\beta \sum_{i} \sigma_{i}^{2}(g) / 2} g|0\rangle .
$$

and obtain

$$
\begin{aligned}
S_{\mathrm{VN}}^{(A)} & =-\frac{1}{Z} \sum_{g \in G} e^{\beta \sum_{i} \sigma_{i}^{z}(g)} \\
& \times \log _{2}\left[\frac{\sum_{h \in G_{A}, k \in G_{B}} e^{\beta \sum_{i} \sigma_{i}^{z}(h g k)}}{Z}\right]
\end{aligned}
$$

where $Z=\sum_{g \in G} e^{\beta \sum_{i} \sigma_{i}^{z}(g)}$.

In order to simplify Eq. (37) with the purpose of computing the topological entropy of the system (23), it is convenient to do the following change of variables. Recall that a generic configuration $g|0\rangle$ is uniquely specified by the set of star operators acting on the reference configuration $|0\rangle$, which we chose to be the ferromagnetic state with all the $\sigma$ spins pointing up, modulo the action of the product of all the star operators (which is equal to the identity). Thus, there is a 1to-2 mapping between $G=\{g\}$ and the configuration space $\Theta=\{\boldsymbol{\theta}\}$ of an Ising model with degrees of freedom $\theta_{s}$ living on the sites $s$ of the square lattice, where for example $\theta_{s}=-1$ $(+1)$ means that the corresponding star operator is (not) acting in the associated $g$. Since each $\sigma$ spin can be flipped only by its two neighboring $\theta$ spins, then $\sigma_{i} \equiv \theta_{s} \theta_{s^{\prime}}$, where $i$ labels the bond between the two neighboring sites $\left\langle s, s^{\prime}\right\rangle$, and

$$
\sum_{g \in G} e^{\beta \sum_{i} \sigma_{i}^{z}(g)} \equiv \frac{1}{2} \sum_{\boldsymbol{\theta} \in \Theta} e^{\beta \sum_{\left\langle s, s^{\prime}\right\rangle} \theta_{s} \theta_{s^{\prime}}} .
$$

Notice that, using the above mapping, the GS wavefunction of our model, Eq. (15), can be rewritten as

$$
|G S\rangle=\sum_{\boldsymbol{\theta} \in \Theta} \frac{e^{\beta \sum_{\left\langle s, s^{\prime}\right\rangle} \theta_{s} \theta_{s^{\prime}} / 2}}{\sqrt{Z}} g(\boldsymbol{\theta})|0\rangle,
$$

where $Z=\sum_{\boldsymbol{\theta} \in \Theta} e^{\beta \sum_{\left\langle s, s^{\prime}\right\rangle} \theta_{s} \theta_{s^{\prime}}}$. Thus, all equal-time correlation functions that can be expressed in terms of the $\theta_{s}$ variables are the same as those of a $2 D$ classical Ising model with reduced nearest-neighbor coupling $J / T=\beta$, implying that the critical point of the latter $\beta_{c}=(1 / 2) \ln (\sqrt{2}+1) \simeq$ 0.4406868 corresponds precisely to the critical point of our quantum system. Notice also that the magnetization in the original $\sigma$ spin language is indeed the nearest-neighbor spinspin correlation (i.e., the energy) in the $\theta$ spin language,

$$
\begin{aligned}
m(\beta) & =\frac{1}{N} \sum_{i}\left\langle G S\left|\hat{\sigma}_{i}^{\mathrm{Z}}\right| G S\right\rangle \\
& =\frac{1}{Z} \sum_{\boldsymbol{\theta} \in \Theta} e^{\beta \sum_{\left\langle s, s^{\prime}\right\rangle} \theta_{s} \theta_{s^{\prime}}}\left[\frac{1}{N} \sum_{i} \sigma_{i}^{\mathrm{Z}}(g(\boldsymbol{\theta}))\right] \\
& =\frac{1}{Z} \sum_{\boldsymbol{\theta} \in \Theta} e^{\beta \sum_{\left\langle s, s^{\prime}\right\rangle} \theta_{s} \theta_{s^{\prime}}}\left[\frac{1}{N} \sum_{\left\langle s, s^{\prime}\right\rangle} \theta_{s} \theta_{s^{\prime}}\right] \\
& =\frac{1}{N} E_{\text {Ising }}(\beta) .
\end{aligned}
$$


Therefore, one concludes that the magnetization $m(\beta)$ is continuous across the transition at $\beta_{c}$ but there is a singularity in its first derivative

$$
\frac{\partial m}{\partial \beta}=\frac{1}{N} \frac{\partial E_{\text {Ising }}}{\partial \beta}=-\beta^{2} \frac{1}{N} C_{\text {Ising }}(\beta),
$$

as the Ising model heat capacity $C_{\text {Ising }}$ diverges logarithmically at $\beta_{c}$.

In the following, we will show how such continuous phase transition is accompanied by a sudden, discontinuous vanishing of the topological entropy of the system.

The case of a configuration of the form $h g k$, with $h \in G_{A}$ and $k \in G_{B}$ requires a few additional steps. First of all, notice that the composition of any two elements $g, \tilde{g} \in G$ is represented in the $\theta$ spin language by the site-by-site product of the two configurations corresponding to $g$ and $\tilde{g}$, respectively: $\theta_{s}(g \tilde{g})=\theta_{s}(g) \theta_{s}(\tilde{g})$. In particular, $\theta_{s}(h g k)=\theta_{s}(h k) \theta_{s}(g)$.

Moreover, using similar arguments as in Ref. 15, the star operators of a bipartite system $(A, B)$ can be divided into bulk star operators, i.e., those acting solely on subsystem $A$ or subsystem $B$, and boundary star operators acting simultaneously on $A$ and $B$ spins. The boundary star operators can be further subdivided into different sets according to the different boundaries around each connected component of $A$ and $B$ (for a total of $m_{A}+m_{B}-1$ boundaries, $m_{A}$ and $m_{B}$ being the number of connected components of $A$ and $B$, respectively).

Let us define a collective operation as the product of all the star operators in one of these sets. That is, the product of all the stars around a connected boundary of the bipartition $(A, B)$. Clearly, the number of such collective operations is given by the number of sets, $m_{A}+m_{B}-1$.

One can show that the subgroup $G_{A} G_{B} \subset G$, to which the product $h k$ belongs, can be generated by all the bulk star operators together with all but one of the collective operators (all but one, independently of which one is chosen to be left out, is required because the product of all boundary star operators is equivalent to the product of all bulk star operators). For example, $G_{A} G_{B}$ is generated by the bulk star operators alone in bipartitions 2 and 3 in Fig. 2, while the product of all boundary star operators along one of the two boundaries must be included to generate $G_{A} G_{B}$ for bipartitions 1 and 4 .

Let us define $\Theta^{b}=\left\{\boldsymbol{\theta}^{b}\right\}$, ' $b$ ' for 'bulk', to be the set of Ising spin configurations on the sites of the square lattice where all $\theta_{s}^{b}$ corresponding to boundary sites $s$ are fixed to equal +1 . Let us also define $\Theta^{\delta}=\left\{\boldsymbol{\theta}^{\delta}\right\}$, ' $\delta$ ' for 'boundary', to be the set of Ising configurations where $\theta_{s}^{\delta}=+1$ for all bulk star operators, $\theta_{s}^{\delta}=+1$ for all boundary star operators belonging to one chosen boundary, and $\theta_{s}^{\delta}= \pm 1$ for the remaining boundary star operators, so long as all $\theta_{s}^{\delta}$ spins belonging to the same boundary have the same sign. Notice that $\Theta^{\delta}=\{\mathbf{1}\}$ for bipartitions 2 and 3 in Fig. 2, where 1 is the configuration with all the spins $\theta_{s}^{\delta}=+1$. One can finally show that there is a one-to-one correspondence between the elements of $G_{A} G_{B}$ and the Ising configurations in $\left\{\boldsymbol{\theta}^{b} \boldsymbol{\theta}^{\delta}, \forall \boldsymbol{\theta}^{b} \in \Theta^{b}, \boldsymbol{\theta}^{\delta} \in \Theta^{\delta}\right\}$, where $\boldsymbol{\theta}^{b} \boldsymbol{\theta}^{\delta}$ represents the site-by-site product of the two configurations (i.e., $\left(\theta^{b} \theta^{\delta}\right)_{s}=\theta_{s}^{b} \theta_{s}^{\delta}$ ). Therefore,

$$
\begin{aligned}
& \sum_{h \in G_{A}, k \in G_{B}} e^{\beta \sum_{i} \sigma_{i}^{z}(h g k)} \equiv \\
& \equiv \sum_{\boldsymbol{\theta}^{b} \in \Theta^{b}, \boldsymbol{\theta}^{\delta} \in \Theta^{\delta}} e^{\beta \sum_{\left\langle s, s^{\prime}\right\rangle} \theta_{s}^{b} \theta_{s}^{\delta} \theta_{s}(g) \theta_{s^{\prime}}(g) \theta_{s^{\prime}}^{\delta} \theta_{s^{\prime}}^{b},}
\end{aligned}
$$

and in particular,

$$
\begin{aligned}
\sum_{\left\langle s, s^{\prime}\right\rangle} \theta_{s}^{b} \theta_{s}^{\delta} \theta_{s}(g) \theta_{s^{\prime}}(g) \theta_{s^{\prime}}^{\delta} \theta_{s^{\prime}}^{b}= & \\
= & \sum_{\left\langle s, s^{\prime}\right\rangle}^{s, \text { bulk }} \theta_{s}^{b} \theta_{s}(g) \theta_{s^{\prime}}(g) \theta_{s^{\prime}}^{b} \\
& +\sum_{s^{\prime} \text { boundary }}^{s \text { bulk }} \theta_{s}^{b} \theta_{s}(g) \theta_{s^{\prime}}(g) \theta_{s^{\prime}}^{\delta} \\
& +\sum_{s, s^{\prime} \text { different boundaries }}^{\left\langle s, s^{\prime}\right\rangle} \theta_{s}^{\delta} \theta_{s}(g) \theta_{s^{\prime}}(g) \theta_{s^{\prime}}^{\delta} \\
& +\sum_{s, s^{\prime} \text { same boundary }}^{\left\langle s, s^{\prime}\right\rangle} \theta_{s}(g) \theta_{s^{\prime}}(g),
\end{aligned}
$$

where we used the fact that if $s$ is in the bulk then $\theta_{s}^{\delta}=+1$, if $s$ belongs to a boundary then $\theta_{s}^{b}=+1$, and if both $s$ and $s^{\prime}$ belong to the same boundary then $\theta_{s}^{\delta} \theta_{s^{\prime}}^{\delta}=+1$.

Let us focus on the bipartitions of interest to compute the topological entropy (23). First of all, in the limit $r, R \rightarrow \infty$ there are no nearest-neighboring stars $s$ and $s^{\prime}$ belonging to two different boundaries. Therefore, the term (45) vanishes identically. For bipartitions 2 and $3, \Theta^{\delta}=\{\mathbf{1}\}$ and

$$
\sum_{\substack{h \in G_{A} \\ k \in G_{B}}} e^{\beta \sum_{i} \sigma_{i}^{\mathrm{z}}(h g k)} \equiv e^{\beta \sum_{\left\langle s, s^{\prime}\right\rangle}^{s, s^{\prime} \text { boundary }} \theta_{s}(g) \theta_{s^{\prime}}(g)} \sum_{\boldsymbol{\theta}^{b} \in \Theta^{b}} e^{\beta \sum_{\left\langle s, s^{\prime}\right\rangle}^{s, s^{\prime} \text { bulk }} \theta_{s}^{b} \theta_{s}(g) \theta_{s^{\prime}}(g) \theta_{s^{\prime}}^{b}} e^{\beta \sum_{\left\langle s, s^{\prime}\right\rangle}^{s^{\prime} \text { boundary }, s \text { bulk }} \theta_{s}^{b} \theta_{s}(g) \theta_{s^{\prime}}(g)} .
$$

The r.h.s. of the above equation can be interpreted as the

partition function of an Ising model with nearest-neighbor 
interactions, where only the bulk degrees of freedom are allowed to flip starting from a given configuration $\boldsymbol{\theta}(g)$. Clearly such partition function is invariant upon changing the initial configuration as long as the new one is in the same ergodic sector.
For example, one can equivalently choose

$$
\tilde{\boldsymbol{\theta}}(g)= \begin{cases}+1 & \text { if } s \text { belongs to the bulk } \\ \theta_{s}(g) & \text { if } s \text { belongs to the boundary }\end{cases}
$$

and the expression above simplifies to

$$
\sum_{\substack{h \in G_{A} \\ k \in G_{B}}} e^{\beta \sum_{i} \sigma_{i}^{\mathrm{z}}(h g k)} \equiv e^{\beta \sum_{\left\langle s, s^{\prime}\right\rangle}^{s, s^{\prime} \text { boundary }} \theta_{s}(g) \theta_{s^{\prime}}(g)} \sum_{\boldsymbol{\theta}^{b} \in \Theta^{b}} e^{\beta \sum_{\left\langle s, s^{\prime}\right\rangle}^{s, s^{\prime} \text { bulk }} \theta_{s}^{b} \theta_{s^{\prime}}^{b}} e^{\beta \sum_{\left\langle s, s^{\prime}\right\rangle}^{s^{\prime} \text { boundary }, s \text { bulk }} \theta_{s}^{b} \theta_{s^{\prime}}(g)}=Z_{2,3}^{\partial}(g) .
$$

Here $Z_{2,3}^{\partial}(g)$ represents the partition function of an Ising model with nearest-neighbor interaction of reduced strength $J / T=\beta$, and with fixed spins along the boundary of bipartitions 2 and 3 , respectively. The values of the spins at the boundary are determined by $g$.
For bipartitions 1 and $4, \Theta^{\delta}=\{\mathbf{1}, \mathbf{f}\}$, where the configuration $\mathbf{f}$ has all the spins equal to +1 except for those belonging to the chosen boundary, say boundary 2 in Fig. 2, which are equal to -1 . In this case

$$
\begin{aligned}
\sum_{\substack{h \in G_{A} \\
k \in G_{B}}} e^{\beta \sum_{i} \sigma_{i}^{\mathrm{z}}(h g k)} & \equiv e^{\beta \sum_{\left\langle s, s^{\prime}\right\rangle}^{s, s^{\prime} \text { same boundary }} \theta_{s}(g) \theta_{s^{\prime}}(g)} \sum_{\boldsymbol{\theta}^{b} \in \Theta^{b}} e^{\beta \sum_{\left\langle s, s^{\prime}\right\rangle}^{s, s^{\prime} \text { bulk }} \theta_{s}^{b} \theta_{s^{\prime}}^{b}} e^{\beta \sum_{\left\langle s, s^{\prime}\right\rangle}^{s^{\prime} \text { boundary 1,s bulk }} \theta_{s}^{b} \theta_{s^{\prime}}(g)} \\
& \times\left(e^{\beta \sum_{\left\langle s, s^{\prime}\right\rangle}^{s^{\prime} \text { boundary 2,s bulk }} \theta_{s}^{b} \theta_{s^{\prime}}(g)}+e^{-\beta \sum_{\left\langle s, s^{\prime}\right\rangle}^{s^{\prime} \text { boundary } 2, s \text { bulk }} \theta_{s}^{b} \theta_{s^{\prime}}(g)}\right) \\
& =Z_{1,4}^{\partial}(g)+Z_{1,4}^{\partial, \text { twisted }}(g) .
\end{aligned}
$$

Here $Z_{1,4}^{\partial}(g)$ are the analog of $Z_{2,3}^{\partial}(g)$ for bipartitions 1 and 4 , respectively, while $Z_{1,4}^{\partial \text {, twisted }}(g)$ differ from the former by the fact that all the (fixed) spins belonging to boundary 2 in bipartitions 1 and 4 respectively have been flipped. In other words, $Z_{1,4}^{\partial}(g)$ represents the partition function of an Ising model with nearest-neighbor interaction of reduced strength $J / T=\beta$, and with fixed spins along the bound- ary of bipartitions 1 and 4 , respectively. The partition functions $Z_{1,4}^{\partial \text {, twisted }}(g)$ differ in that the spins along one of the two boundaries have been flipped with respect to their values in $Z_{1,4}^{\partial}(g)$. Again, the values of the spins at the boundary are determined by $g$.

In this notation, the topological entropy of the system can be written as

$$
S_{\text {topo }}=\lim _{r, R \rightarrow \infty}\left\{\frac{1}{Z} \sum_{g \in G} e^{\beta \sum_{i} \sigma_{i}^{z}(g)} \log _{2} \frac{\left[Z_{1}^{\partial}(g)+Z_{1}^{\partial, \text { twisted }}(g)\right]\left[Z_{4}^{\partial}(g)+Z_{4}^{\partial, \text { twisted }}(g)\right]}{Z_{2}^{\partial}(g) Z_{3}^{\partial}(g)}\right\}
$$

where the sum over $g$ acts as a weighed average of the logarithmic term over all possible values of the spins at the boundary. Notice that in Eq. (51) the partitions with two boundaries, and hence with non-trivial topology, are those that appear with two contributions (bipartitions 1 and 4), corresponding to some relative boundary conditions (BCs) and their twisted counterparts. These contributions, as we show below in detail, are responsible for the non-vanishing topological entropy. 
In the topological phase, the two partition functions for the twisted and untwisted BCs contribute equally, and in the nontopological phase, one partition function is exponentially suppressed when compared to the other, in the thermodynamic limit. Therefore, there is an extra entropy contribution in one of the phases depending on whether the boundaries of topologically non-trivial bipartitions are twisted or not relative to one another.

From Eq. (51), the behavior of the topological entropy can be qualitatively argued as follows. Deep in the disordered phase, where the correlations are short ranged, the choice of boundary conditions is likely to affect the partition function of the system only with exponentially small corrections. Thus, we can expect to have $Z_{1}^{\partial}(g) Z_{4}^{\partial}(g) \simeq Z_{1}^{\partial \text {, twisted }}(g) Z_{4}^{\partial}(g) \simeq$ $\ldots \simeq Z_{2}^{\partial}(g) Z_{3}^{\partial}(g)$ and $S_{\text {topo }}=2$. On the other hand, deep in the (ferromagnetically) ordered phase the partition function of a system with twisted boundary conditions is exponentially suppressed with respect to the one without the twist. Thus, $Z_{1}^{\partial}(g) \gg Z_{1}^{\partial \text {, twisted }}(g), Z_{4}^{\partial}(g) \gg Z_{4}^{\partial \text {, twisted }}(g)$, while $Z_{1}^{\partial}(g) Z_{4}^{\partial}(g) \simeq Z_{2}^{\partial}(g) Z_{3}^{\partial}(g)$ still holds. This leads to $S_{\text {topo }}=0$.

In the following two sections we will show with rigorous arguments that the behavior of the topological entropy across the transition is strongly first order, with a sudden jump from $S_{\text {topo }}=2$ to $S_{\text {topo }}=0$.

\section{The disordered phase $\left(\beta<\beta_{c}\right)$}

In the limit of small $\beta$, namely above the ordering transition, one can compute $S_{\text {topo }}$ via the high-temperature expansion of the Ising model with fixed spins at the boundary.

Let us rewrite,

$$
\begin{aligned}
Z_{2}^{\partial}(g) & =\sum_{\boldsymbol{\theta}^{b} \in \Theta^{b}} \prod_{\text {bonds } i}\left(\cosh \beta+\sinh \beta \theta_{s_{i}} \theta_{s_{i}^{\prime}}\right) \\
& =2^{N / 2-\delta} c^{N} \sum_{\mathcal{G}} t^{\ell(\mathcal{G})} \prod_{s \in \mathcal{E}_{\mathcal{G}}} \theta_{s}(g),
\end{aligned}
$$

where $s_{i}, s_{i}^{\prime}$ are the sites at the ends of bond $i, N$ is the total number of bonds on the lattice, $\delta$ is the length of the boundary in number of $\theta$ spins, $c=\cosh \beta, t=\tanh \beta$. The sum over $\mathcal{G}$ runs over all possible graphs on the bonds of the square lattice, composed entirely of closed loops and open strings connecting two boundary spins. The product $\prod_{s \in \mathcal{E}_{\mathcal{G}}} \theta_{s}(g)$ encompasses all the boundary spins that appear as end points (the set $\mathcal{E}_{\mathcal{G}}$ ) of open strings in $\mathcal{G}$. Finally, $\ell(\mathcal{G})$ is the total length of the closed loops and open strings in $\mathcal{G}$.

Analogously for $Z_{3}^{\partial}(g)$. The case of $Z_{1}^{\partial}(g)$ and $Z_{4}^{\partial}(g)$ differs from $Z_{2}^{\partial}(g)$ and $Z_{3}^{\partial}(g)$ in that there are now two types of open strings: those going from one boundary to itself and those connecting the two boundaries (see Fig. 2).

Next, let us compare the product $Z_{2}^{\partial}(g) Z_{3}^{\partial}(g)$ with the product $Z_{1}^{\partial}(g) Z_{4}^{\partial}(g)$. Notice that bipartitions 2 and 3 have precisely the same total combined boundary as bipartitions 1 and 4 . In order for a graph to appear in one of the two products and not in the other, it needs to comprise loops or strings that are able to tell the difference between to two possible origins $(2+3$ vs. $1+4)$ of the total combined boundary.

Examples of such open strings are shown in Fig. 3. One
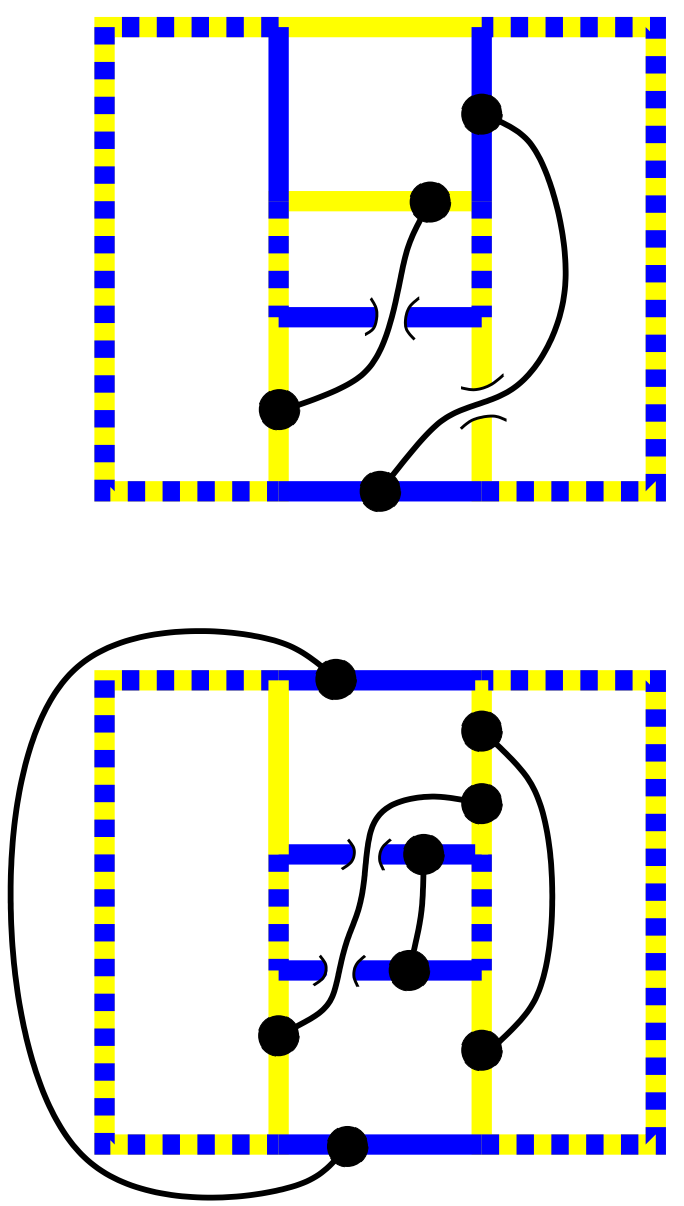

FIG. 3: (Color online) - Examples of open strings that appear in the expansion of $Z_{2}^{\partial}(g) Z_{3}^{\partial}(g)$ but are not present in the expansion of $Z_{1}^{\partial}(g) Z_{4}^{\partial}(g)$ (Top), and vice versa (Bottom). Top panel: The thick yellow and blue lines correspond to the boundaries in bipartitions 2 and 3 respectively. Boundaries belonging to both are shown in a thick dashed yellow-blue pattern. The strings in question are symbolically represented by thin black lines. Bottom panel: Same color coding, with yellow corresponding to bipartition 4 and blue corresponding to biparition 1. Notice that strings appearing in one expansion and not in the other must connect boundaries of the same solid color, and therefore cannot be shorter than $R-2 r$.

can show that these telltale strings, and the analogous closed loops, cannot be arbitrarily short, and their length is bounded from below by $R-2 r$. As a consequence, the corresponding graphs are exponentially suppressed at least as $t^{R-2 r}$, and in the limit $r, R \rightarrow \infty$ with $R-2 r \rightarrow \infty$, implicit in the definition of the topological entropy, one obtains

$$
\frac{Z_{1}^{\partial}(g) Z_{4}^{\partial}(g)}{Z_{2}^{\partial}(g) Z_{3}^{\partial}(g)} \rightarrow 1
$$


Similar considerations apply when comparing the product $Z_{1}^{\partial}(g) Z_{4}^{\partial}(g)$ with products of the kind $Z_{1}^{\partial \text {, twisted }}(g) Z_{4}^{\partial}(g)$. In this case, the boundaries involved are exaclty the same, and the relevant telltale elements of the graph are open strings connecting one of the two components of the boundary with the other. Such strings are in fact the only elements that are sensitive to the twisted boundary conditions. Clearly the length of these strings is bounded from below by $R-2 r$, and

$$
\frac{Z_{1}^{\partial, \text { twisted }}(g) Z_{4}^{\partial}(g)}{Z_{1}^{\partial}(g) Z_{4}^{\partial}(g)} \rightarrow 1
$$

exponentially fast, at least as $t^{R-2 r}$, with $(R-2 r) \rightarrow \infty$.

Of course, our reasoning is correct up to the point where the high temperature expansion breaks down, and entropic contributions balance the exponential suppression. Said differently, this is the case when the correlation length in the Ising model goes to infinity, and the large $r, R$ limit does not guarantee that the ratios of products of partition functions above tend to one.

Given Eqs. (53)54), we can finally use Eq. (51) to obtain the topological entropy of the system throughout the disordered phase $\beta<\beta_{c} \simeq 0.4406868$,

$$
\begin{aligned}
S_{\text {topo }} & =\frac{1}{Z} \sum_{g \in G} e^{\beta \sum_{i} \sigma_{i}^{z}(g)} \log _{2} 4 \\
& =\log _{2} 4=2 .
\end{aligned}
$$

\section{2. $\quad$ The Landau-Ginzburg ordered phase $\left(\beta>\beta_{c}\right)$}

What happens below this transition? Rather than attempting a low-temperature expansion, it is convenient to use the duality relations derived by A. Bugrij and V. Shadura in Ref. 16 for the inhomogeneous, finite-size Ising model. In particular, they obtained the duality relations for a square lattice Ising model wrapped around a cylinder of finite length, with fixed, free and mixed boundary conditions. Following the usual convention, let us label $\tilde{\beta}$ the coupling constant of the dual Ising model (defined on the plaquettes of the original lattice), which is related to $\beta$ by the duality relation $\sinh \beta \sinh \tilde{\beta}=1$. Let us also indicate with $Z\left(\delta, \delta^{\prime}\right)$ and $\tilde{Z}\left(\delta, \delta^{\prime}\right)$ the partition functions of the system on the finite cylinder and its dual, with $\delta, \delta^{\prime}$ specifying the boundary conditions, namely $\delta, \delta^{\prime}=\bigcirc, \times$ for free and fixed boundary spins, respectively. With this notation in mind, the results by Bugrij and Shadura - to the purpose of the present paper - can be summarized by 16

$$
\begin{aligned}
& \tilde{Z}(\bigcirc, \bigcirc)=\mathcal{K}\left[Z(\times, \times)+Z^{\text {twisted }}(\times, \times)\right] \\
& \tilde{Z}(\times, \bigcirc)=\mathcal{K} Z(\bigcirc, \times) \\
& \tilde{Z}(\bigcirc, \times)=\mathcal{K} Z(\times, \bigcirc),
\end{aligned}
$$

where $Z^{\text {twisted }}(\times, \times)$ differs from $Z(\times, \times)$ by the fact that the fixed boundary spins at one end of the cylinder have been flipped. Notice that the proportionality coefficient $\mathcal{K}$ is the same in all the equations, and that $Z(\bigcirc, \times)=Z(\times, \bigcirc)$ and $\tilde{Z}(\times, \bigcirc)=\tilde{Z}(\bigcirc, \times)$.
Let us then consider $Z_{2}^{\partial}$ in Eq. (49). Thanks to the nearestneighbor character of the interaction between $\theta$ spins, subsystem $A$ interacts only with itself and with the boundary $\delta_{2}$, and so does subsystem $B$. Thus, one can factorize the two subsystems and obtain (cfr. Eq.(49))

$$
\begin{aligned}
& \sum_{h \in G_{2 A}} e^{\beta \sum_{i} \sigma_{i}^{z}(h g k)} \equiv e^{\beta \sum_{\left\langle s, s^{\prime}\right\rangle}^{s, s^{\prime} \in \delta_{2}} \theta_{s}(g) \theta_{s^{\prime}}(g)} \\
& \times\left(\sum_{\boldsymbol{\theta}_{2 A}^{b} \in \Theta_{2 A}^{b}} e^{\beta \sum_{\left\langle s, s^{\prime}\right\rangle}^{s, s^{\prime} \text { bulk }} \theta_{s^{b}}^{b} \theta_{s^{\prime}}^{b}} e^{\beta \sum_{\left\langle s, s^{\prime}\right\rangle}^{s^{\prime} \text { boundry,s bulk }} \theta_{s}^{b} \theta_{s^{\prime}}(g)}\right) \\
& \times\left(\sum_{\boldsymbol{\theta}_{2 B}^{b} \in \Theta_{2 B}^{b}} e^{\beta \sum_{\left\langle s, s^{\prime}\right\rangle}^{s, s^{\prime} \text { bulk }} \theta_{s}^{b} \theta_{s^{\prime}}^{b}} e^{\beta \sum_{\left\langle s, s^{\prime}\right\rangle}^{s^{\prime} \text { boundary,s bulk }} \theta_{s^{b}}^{b} \theta_{s^{\prime}}(g)}\right) \\
& =\left(e^{\beta \sum_{\left\langle s, s^{\prime}\right\rangle}^{s, s^{\prime} \delta_{2}} \theta_{s}(g) \theta_{s^{\prime}}(g)}\right) Z_{2 A}^{\partial}(g) Z_{2 B}^{\partial}(g) .
\end{aligned}
$$

Similar arguments apply to bipartition 3,

$$
\begin{aligned}
& \sum_{\substack{h \in G_{3 A} \\
k \in G_{3 B}}} e^{\beta \sum_{i} \sigma_{i}^{z}(h g k)}= \\
& \quad=\left(e^{\beta \sum_{\left\langle s, s^{\prime}\right\rangle}^{s, s^{\prime} \in \delta_{3}} \theta_{s}(g) \theta_{s^{\prime}}(g)}\right) Z_{3 A}^{\partial}(g) Z_{3 B}^{\partial}(g),
\end{aligned}
$$

and with a few more steps, to bipartitions 1 and 4 as well (cfr. Eq.(50),

$$
\begin{aligned}
\sum_{\substack{h \in G_{1 A} \\
k \in G_{1 B}}} e^{\beta \sum_{i} \sigma_{i}^{z}(h g k)}= \\
=\left(e^{\beta \sum_{\left\langle s, s^{\prime}\right\rangle}^{s, s^{\prime} \in \delta_{1}, \text { boundary } 1} \theta_{s}(g) \theta_{s^{\prime}}(g)}\right) \\
\times\left(e^{\beta \sum_{\left\langle s, s^{\prime}\right\rangle}^{s, s^{\prime} \in \delta_{1}, \text { boundary } 2} \theta_{s}(g) \theta_{s^{\prime}}(g)}\right) \\
\times\left[Z_{1 A}^{\partial}(g)+Z_{1 A}^{\partial, \text { twisted }}(g)\right] Z_{1 B_{1}}^{\partial}(g) Z_{1 B_{2}}^{\partial}(g),
\end{aligned}
$$

and

$$
\begin{aligned}
& \sum_{\substack{h \in G_{4 A} \\
k \in G_{4 B}}} e^{\beta \sum_{i} \sigma_{i}^{\mathrm{z}}(h g k)}= \\
&=\left(e^{\beta \sum_{\left\langle s, s^{\prime}\right\rangle}^{s, s^{\prime}, \delta_{4}, \text { boundary 1 }} \theta_{s}(g) \theta_{s^{\prime}}(g)}\right) \\
& \times\left(e^{\beta \sum_{\left\langle s, s^{\prime}\right\rangle}^{s, s^{\prime} \in \delta_{4}, \text { boundary 2 }} \theta_{s}(g) \theta_{s^{\prime}}(g)}\right) \\
& \times Z_{4 A_{1}}^{\partial}(g) Z_{4 A_{2}}^{\partial}(g)\left[Z_{4 B}^{\partial}(g)+Z_{4 B}^{\partial, \text { twisted }}(g)\right],
\end{aligned}
$$


where $1 B_{1}$ and $1 B_{2}$ refer to the two connected components of subsystem $B$ in bipartition 1, i.e., the component inside boundary 1 and the component outside boundary 2 , and analogously for $4 A_{1}$ and $4 A_{2}$.

In order to apply Eqs. (55) 577) to the present case, some further considerations on the bipartitions in Fig. 2 are needed. Recall that, although $S_{\text {topo }}$ is indeed a quantity of order one, we expressed it in Eq. (51) in terms of a ratio of extensive partition functions $Z_{i}^{\partial}(g)$. Thus, any sub-extensive correction to these partition functions (i.e., $\mathcal{O}\left(2^{N^{\alpha}}\right)$, with $\alpha<1, N$ being the number of degrees of freedom in the system) will only amount to an exponentially small correction to $S_{\text {topo }}$, that vanishes in the thermodynamic limit. In this context, the partition function $Z_{1 A}^{\partial}(g)$ (see Fig. (4) is 'equivalent', in the thermodynamic limit, to the partition function of an Ising model on an infinite cylinder with fixed boundaries at the edges (boundary 1 and 2, respectively). Similarly, the partition function $Z_{1 B_{1}}^{\partial}(g)$ can be regarded as that of an Ising model on an infinite cylinder with fixed boundary conditions on one edge (boundary 1) and open boundary conditions on a suitably introduced boundary $\gamma_{1}$. Finally, the same approach can be used for $Z_{1 B_{2}}^{\partial}(g)$, with fixed boundary conditions on one edge (boundary 2) and open boundary conditions on another suitably introduced boundary $\gamma_{4}$. Qualitatively, this is illustrated in Fig. 4 h, where the spins on boundary 1 and boundary 2 are fixed and those belonging to $\gamma_{1}$ and $\gamma_{4}$ are free. Notice that the

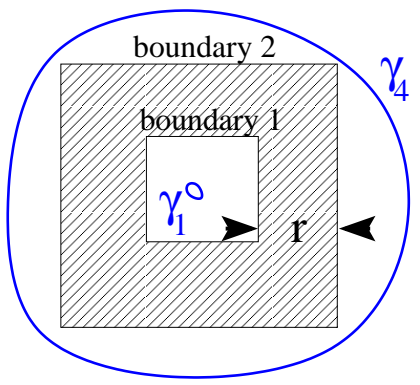

(a)

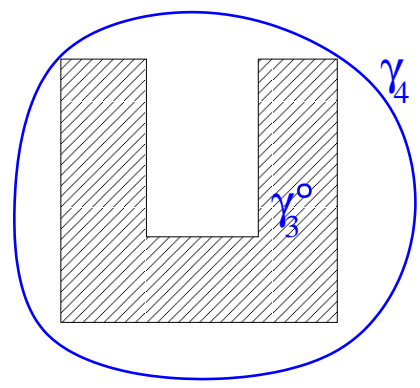

(c)

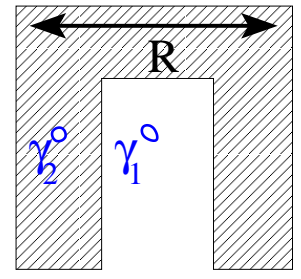

(b)

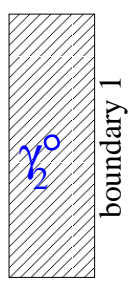

(d)
FIG. 4: (Color online) - Illustration of the four bipartitions used to compute the topological entropy in Ref. 10, with a possible choice for the additional boundaries needed to map each partition onto an infinite cylinder in the thermodynamic limit. mapping onto infinite cylinders requires the distance between any of the $\gamma_{i}$ boundaries introduced in Fig. 4 and any of the original boundaries in Fig. 2 2 to diverge with system size. Similar arguments apply to bipartitions 2, 3 and 4 . This leads to a correspondence between our factorized partition functions and those used in $\operatorname{Ref} 16$, namely

$$
\begin{aligned}
Z_{1 B_{1}}^{\partial}(g), Z_{1 B_{2}}^{\partial}(g), Z_{2 A}^{\partial}(g), & \\
Z_{2 B}^{\partial}(g), Z_{3 A}^{\partial}(g), Z_{3 B}^{\partial}(g), & \\
Z_{4 A_{1}}^{\partial}(g), Z_{4 A_{2}}^{\partial}(g) & \sim Z(\bigcirc, \times) \\
Z_{1 A}^{\partial}(g), Z_{4 B}^{\partial}(g) & \sim Z(\times, \times) \\
Z_{1 A}^{\partial, \text { twisted }}(g), Z_{4 B}^{\partial \text {,twsted }}(g) & \sim Z^{\text {twisted }}(\times, \times) .
\end{aligned}
$$

The results in Ref. 16 can then be applied to our systems and lead to the following equations:

$$
\begin{aligned}
\tilde{Z}_{1 A}^{\partial}(\bigcirc, \bigcirc) & \propto Z_{1 A}^{\partial}(g)+Z_{1 A}^{\partial, \text { twisted }}(g) \\
\tilde{Z}_{1 B_{1}}^{\partial}(\times, \bigcirc) & \propto Z_{1 B_{1}}^{\partial}(g) \\
\tilde{Z}_{1 B_{2}}^{\partial}(\times, \bigcirc) & \propto Z_{1 B_{2}}^{\partial}(g)
\end{aligned}
$$

$$
\begin{aligned}
& \tilde{Z}_{2 A}^{\partial}(\times, \bigcirc) \propto Z_{2 A}^{\partial}(g) \\
& \tilde{Z}_{2 B}^{\partial}(\times, \bigcirc) \propto Z_{2 B}^{\partial}(g)
\end{aligned}
$$

$$
\begin{aligned}
& \tilde{Z}_{3 A}^{\partial}(\times, \bigcirc) \propto Z_{3 A}^{\partial}(g) \\
& \tilde{Z}_{3 B}^{\partial}(\times, \bigcirc) \propto Z_{3 B}^{\partial}(g)
\end{aligned}
$$

$$
\begin{aligned}
\tilde{Z}_{4 B}^{\partial}(\bigcirc, \bigcirc) & \propto Z_{4 B}^{\partial}(g)+Z_{4 B}^{\partial, \text { twisted }}(g) \\
\tilde{Z}_{4 A_{1}}^{\partial}(\times, \bigcirc) & \propto Z_{4 A_{1}}^{\partial}(g) \\
\tilde{Z}_{4 A_{2}}^{\partial}(\times, \bigcirc) & \propto Z_{4 A_{2}}^{\partial}(g) .
\end{aligned}
$$

For convenience of notation, let us define the dual partition functions for the whole system in the different bipartitions

$$
\begin{aligned}
\tilde{Z}_{1}^{\partial} & \equiv \tilde{Z}_{1 A}^{\partial}(\bigcirc, \bigcirc) \tilde{Z}_{1 B_{1}}^{\partial}(\times, \bigcirc) \tilde{Z}_{1 B_{2}}^{\partial}(\times, \bigcirc) \\
\tilde{Z}_{2}^{\partial} & \equiv \tilde{Z}_{2 A}^{\partial}(\times, \bigcirc) \tilde{Z}_{2 B}^{\partial}(\times, \bigcirc) \\
\tilde{Z}_{3}^{\partial} & \equiv \tilde{Z}_{3 A}^{\partial}(\times, \bigcirc) \tilde{Z}_{3 B}^{\partial}(\times, \bigcirc) \\
\tilde{Z}_{4}^{\partial} & \equiv \tilde{Z}_{4 A_{1}}^{\partial}(\times, \bigcirc) \tilde{Z}_{4 A_{2}}^{\partial}(\times, \bigcirc) \tilde{Z}_{4 B}^{\partial}(\bigcirc, \bigcirc)
\end{aligned}
$$

Finally, we have all the ingredients to evaluate the topological entropy for $\beta>\beta_{c}$. Let us first rewrite Eq. (51) using Eqs. (58,61) instead of Eqs. (49,50): 


$$
\begin{aligned}
S_{\text {topo }}=\lim _{r, R \rightarrow \infty}\{ & \frac{1}{Z} \sum_{g \in G} e^{\beta \sum_{i} \sigma_{i}^{z}(g)} \\
& \times\left[\log _{2} \frac{\left[Z_{1 A}^{\partial}(g)+Z_{1 A}^{\partial, \text { twisted }}(g)\right] Z_{1 B_{1}}^{\partial}(g) Z_{1 B_{2}}^{\partial}(g) Z_{4 A_{1}}^{\partial}(g) Z_{4 A_{2}}^{\partial}(g)\left[Z_{4 B}^{\partial}(g)+Z_{4 B}^{\partial, \text { twisted }}(g)\right]}{Z_{2 A}^{\partial}(g) Z_{2 B}^{\partial}(g) Z_{3 A}^{\partial}(g) Z_{3 B}^{\partial}(g)}\right. \\
& \left.\left.+\sum_{\left\langle s, s^{\prime}\right\rangle}^{s, s^{\prime} \in \delta_{1}+\delta_{4}} \theta_{s}(g) \theta_{s^{\prime}}(g)-\sum_{\left\langle s, s^{\prime}\right\rangle}^{s, s^{\prime} \in \delta_{2}+\delta_{3}} \theta_{s}(g) \theta_{s^{\prime}}(g)\right]\right\},
\end{aligned}
$$

where the last two terms inside the square brackets come from the exponential factors in Eqs. (58,61). Using the duality relations (62), we can identify

$$
\begin{gathered}
\frac{\left[Z_{1 A}^{\partial}(g)+Z_{1 A}^{\partial, \text { twisted }}(g)\right] Z_{1 B_{1}}^{\partial}(g) Z_{1 B_{2}}^{\partial}(g) Z_{4 A_{1}}^{\partial}(g) Z_{4 A_{2}}^{\partial}(g)\left[Z_{4 B}^{\partial}(g)+Z_{4 B}^{\partial, \text { twisted }}(g)\right]}{Z_{2 A}^{\partial}(g) Z_{2 B}^{\partial}(g) Z_{3 A}^{\partial}(g) Z_{3 B}^{\partial}(g)}= \\
=\frac{\tilde{Z}_{1 A}^{\partial}(\bigcirc, \bigcirc) \tilde{Z}_{1 B_{1}}^{\partial}(\times, \bigcirc) \tilde{Z}_{1 B_{2}}^{\partial}(\times, \bigcirc) \tilde{Z}_{4 A_{1}}^{\partial}(\times, \bigcirc) \tilde{Z}_{4 A_{2}}^{\partial}(\times, \bigcirc) \tilde{Z}_{4 B}^{\partial}(\bigcirc, \bigcirc)}{\tilde{Z}_{2 A}^{\partial}(\times, \bigcirc) \tilde{Z}_{2 B}^{\partial}(\times, \bigcirc) \tilde{Z}_{3 A}^{\partial}(\times, \bigcirc) \tilde{Z}_{3 B}^{\partial}(\times, \bigcirc)} \\
\equiv \frac{\tilde{Z}_{1}^{\partial} \tilde{Z}_{4}^{\partial}}{\tilde{Z}_{2}^{\partial} \tilde{Z}_{3}^{\partial} .}
\end{gathered}
$$

For $\beta>\beta_{c}$, the dual Ising models are in the disordered phase and one can perform a high-temperature expansion to calculate the ratio $\left(\tilde{Z}_{1}^{\partial} \tilde{Z}_{4}^{\partial}\right) /\left(\tilde{Z}_{2}^{\partial} \tilde{Z}_{3}^{\partial}\right)$. Using the same loop description as for the original system, with $t$ replaced by $\tilde{t}=$ $\tanh (\tilde{\beta})$, one can show that $\left(\tilde{Z}_{1}^{\partial} \tilde{Z}_{4}^{\partial}\right) /\left(\tilde{Z}_{2}^{\partial} \tilde{Z}_{3}^{\partial}\right)=1$ in the thermodynamic limit.

The remaining terms in Eq. (67) can be dealt with more promptly by reverting back to the original $\sigma$ spin degrees of freedom,

$$
\begin{aligned}
\sum_{\left\langle s, s^{\prime}\right\rangle}^{s, s^{\prime} \in \delta_{1}+\delta_{4}} \theta_{s}(g) \theta_{s^{\prime}}(g) & -\sum_{\left\langle s, s^{\prime}\right\rangle}^{s, s^{\prime} \in \delta_{2}+\delta_{3}} \theta_{s}(g) \theta_{s^{\prime}}(g)= \\
& =\sum_{i \in \delta_{1}+\delta_{4}} \sigma_{i}(g)-\sum_{i \in \delta_{2}+\delta_{3}} \sigma_{i}(g)
\end{aligned}
$$

where $i$ labels the bonds of the square lattice, and $i \in \delta$ means that the bond $i$ connects two sites $s$ and $s^{\prime}$ belonging to $\delta$. This contribution can be shown to vanish identically since the set of boundary $\sigma$ spins in bipartitions 1 and 4 is identical to the set of boundary spins in bipartitions 2 and 3 (see Fig. (2)).

In the end we find that

$$
S_{\text {topo }}=\lim _{r, R \rightarrow \infty}\left\{\frac{1}{Z} \sum_{g \in G} e^{\beta \sum_{i} \sigma_{i}^{\mathrm{z}}(g)} \log _{2} \frac{\tilde{Z}_{1}^{\partial} \tilde{Z}_{4}^{\partial}}{\tilde{Z}_{2}^{\partial} \tilde{Z}_{3}^{\partial}}\right\}=0
$$

identically in the ordered phase $\beta>\beta_{c}$.

\section{B. Beyond 1-body potentials}

As we already mentioned, the calculations carried out in Sec. III for the specific model presented in this paper can be straightforwardly extended to the case of any factorizable wavefunction. All one needs to do is identify a proper set of local generators (i.e., acting on the $\sigma$ spins contained within a disc of finite radius) for the group $G$, and the equivalent of the collective boundary flip operators. The rest of the derivation follows essentially unchanged, in the limit $r, R \rightarrow \infty$.

What happens if we attempt to generalize our approach further and we consider non-factorizable wavefunctions? For simplicity, take once again the Kitaev-like GS wavefunction in Eq. (15), but replace the argument of the exponential $\beta \sum_{i} \sigma_{i}^{\mathrm{z}}(g, \alpha) / 2$ with some generic function $-\beta E_{g} / 2$. As we can see immediately from Eq. (24), our approach to compute the topological entropy can no longer be used from the very first stage. On the other hand, it is tempting to conjecture that, so long as $E_{g}$ is short ranged (i.e., it can be written as the sum of terms involving $\sigma$ spins within a disc of finite radius on the lattice), the error that one makes by neglecting the terms involving spins across the boundary of a bipartition $\left(E_{g}^{\partial}\right)$ does not give topological contributions to $S_{\text {topo }}$. Under this assumption, one can then set $E_{g}^{\partial}=0$ and use the approximate equality $E_{g} \simeq E_{g_{A}}^{A}+E_{g_{B}}^{B}$ to re-establish the factorability needed to carry on with the calculations. The result obtained for $S_{\text {topo }}$ in Eq. (51), which employed this approximation, nonetheless shows no explicit dependence on it in the final expression, and one could then reinstate the full $E_{g}$ at that stage. If the conjecture above is correct, the formula in Eq. (51) gives the exact topological entropy for a generic GS wavefunction that satis- 
fies (i) the positive amplitude condition, (ii) the group condition for $G, \frac{17}{, 1}$ and (iii) the locality (i.e., short ranged) condition on $E_{g}$.

A simple example where this conjecture can be applied rather straightforwardly is the case where Eqs. (14) and (15) are replaced by

$$
\begin{aligned}
H & =H_{\text {Kitaev }}+\lambda_{1} \sum_{s} e^{-\beta \sum_{i \in s} \sum_{\langle i j\rangle, j \notin s} \hat{\sigma}_{i}^{z} \hat{\sigma}_{j}^{z}} \\
|G S\rangle & =\frac{1}{\sqrt{Z}} \sum_{g \in G} e^{\beta \sum_{\langle i j\rangle} \sigma_{i}^{z}(g) \sigma_{j}^{2}(g) / 2} g|0\rangle .
\end{aligned}
$$

(For a discussion of the general construction scheme of such type of Hamiltonians, see Ref. 13.) Here $|G S\rangle$ is the GS wavefunction of $H$ in the topological sector where $\prod_{i \in p} \hat{\sigma}_{i}^{z}=+1$. The notation $\langle i j\rangle, j \notin s$ stands for $j$ nearest-neighbor of $i$ but not adjacent to the same vertex $s$. Without loss of generality, we consider the range $\beta \in(0, \infty)$, where the new term in the Hamiltonian favors ferromagnetic order in the $\sigma$ spins.

Let us then introduce the same description in terms of the $\theta$ spins, as in the previous section. Given that the product of two nearest-neighboring $\sigma$ spins translates into the product of two next-nearest-neighboring $\theta$ spins, we obtain

$$
\sum_{g \in G} e^{\beta \sum_{\langle i j\rangle} \sigma_{i}^{z}(g) \sigma_{j}^{z}(g)} \equiv \frac{1}{2} \sum_{\boldsymbol{\theta} \in \Theta} e^{2 \beta \sum_{\left\langle\left\langle s, s^{\prime}\right\rangle\right\rangle} \theta_{s} \theta_{s^{\prime}}}
$$

(notice the additional factor of 2 in the exponent due to the fact that the same product $\theta_{s} \theta_{s^{\prime}}$ corresponds to two distinct products $\left.\sigma_{i}^{\mathrm{Z}} \sigma_{j}^{\mathrm{Z}}\right)$, and

$$
|G S\rangle=\sum_{\boldsymbol{\theta} \in \Theta} \frac{e^{\beta \sum_{\left\langle\left\langle s, s^{\prime}\right\rangle\right\rangle} \theta_{s} \theta_{s^{\prime}}}}{\sqrt{Z}} g(\boldsymbol{\theta})|0\rangle,
$$

where $Z=\sum_{\boldsymbol{\theta} \in \Theta} e^{2 \beta \sum_{\left\langle\left\langle s, s^{\prime}\right\rangle\right\rangle} \theta_{s} \theta_{s^{\prime}}}$. The latter is the partition function of a square-lattice Ising model with sole nextnearest-neighbor interactions, which factorizes into the product of two decoupled Ising models with nearest-neighbor interactions (namely corresponding to the $\theta$ spins on each of the two sublattices). In this case, all equal-time correlators in the GS of the quantum system can be written in terms of classical correlators of two decoupled Ising models. As before, we expect the system to undergo a phase transition when the two Ising models become critical at $\beta_{c}=(1 / 4) \ln (\sqrt{2}+1) \simeq$ 0.2203434 . However, contrarily to the previous case, the new model undergoes a spontaneous symmetry-breaking (LandauGinzburg) phase transition! This is best seen by mapping the system onto a quantum eight-vertex model, as discussed below. The local order parameter that captures the transition is the magnetization of the $\sigma$ spins, whose expectation value can be written as an ensemble average of the product of two neighboring $\theta$ spins, i.e., belonging to two decoupled Ising models. Clearly such average vanishes identically in the hightemperature phase $\beta<\beta_{c}$, while it becomes finite in the ordered phase $\beta>\beta_{c}$. Notice that this local order parameter that acquires an expectation value does so in the nontopologically ordered phase, as expected from the fact that no local order parameter exists that resolves the topological phase.

What is the fate of the topological entropy across this Landau-Ginzburg phase transition? According to the conjecture above, we can directly substitute $\sum_{i} \sigma_{i}^{z} \rightarrow \sum_{\langle i j\rangle} \sigma_{i}^{z} \sigma_{j}^{z}$ into Eq. (51) and compute $S_{\text {topo }}$. This amounts to replacing the boundary Ising partition functions in the argument of the logarithm with the partition functions of two decoupled boundary Ising models. As a result, all calculations carried out in the previous section remain essentially unchanged and one arrives to the identical result that $S_{\text {topo }}=2$ throughout the high-temperature phase, and vanishes otherwise.

This scenario is in agreement with previous results on a quantum version of the eight-vertex model by Ardonne et al.,$\frac{18}{n}$ whose GS is a generalization of the one in our model. Consider indeed the wavefunction in Eq. (69). Given the nature of the group $G$, the four spins belonging to any plaquette of the square lattice can assume only eight distinct configurations $\left(\prod_{i \in p} \hat{\sigma}_{i}^{z}=+1\right)$, illustrated in Fig. 5. Such configurations map naturally onto the vertices of an eight-vertex model upon replacing each positive spin with an arrow along the corresponding bond of the dual lattice, pointing, say, from sublattice $A$ to sublattice $B$, and vice versa for the negative spins (as shown in Fig. 57. Given that $\sum_{\langle i j\rangle} \cdots \equiv \sum_{s} \sum_{\langle i j\rangle \in s} \cdots$, the amplitudes in the GS wavefunction (69) factorize into products of vertex fugacities $\exp \left(\beta \sum_{\langle i j\rangle \in s} \sigma_{i}^{\mathrm{z}} \sigma_{j}^{\mathrm{z}} / 2\right)$. In the notation of Fig. 5, the vertex fugacities assume the values $a=b=$ $1, c=e^{-2 \beta}$ and $d=e^{2 \beta}$. The GS spatial properties of our model are therefore captured by a classical eight-vertex model with the appropriate fugacities ${ }^{18}$ (but see Ref. 13 for a general discussion of such quantum-to-classical correspondence), and one can then use Baxter's exact solution ${ }^{19}$ to obtain the phase diagram as well as the scaling exponents at the critical point. All this is discussed in detail in Ref. 18: the model undergoes a second-order, $\mathbb{Z}_{2}$-symmetry-breaking phase transition when $d^{2}=c^{2}+2$ (i.e., $\beta_{c}=(1 / 4) \ln (\sqrt{2}+1)$ ), separating a topologically ordered liquid phase from a Landau-Ginzburg ordered phase. The local order parameter across the transition is indeed the magnetization in the original $\sigma$ spins.

\section{CONCLUSIONS}

In this paper we studied a topological quantum phase transition in a microscopic model that can be examined analytically. For this system, an extension of the toric code, the ground state wavefunction can be written exactly as a function of the parameter $\beta$ that drives the system across the quantum phase transition. We computed the topological entropy for this system as a function of $\beta$, and showed that it remains at a constant non-zero value throughout the topologically ordered phase $\left(\beta<\beta_{c} \simeq 0.4406868\right)$. Immediately after the quantum phase transition at $\beta_{c}$, the topological entropy drops to zero and remains so in the non-topologically ordered phase $\left(\beta>\beta_{c}\right)$.

The GS wavefunction of our quantum system has positive amplitudes in the basis of choice. This property allows us to relate many quantities that are relevant in characterizing 

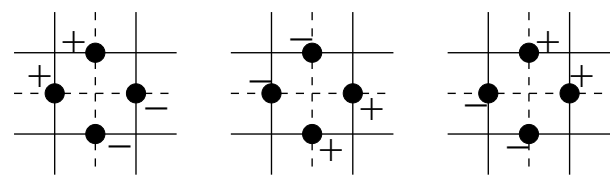

a
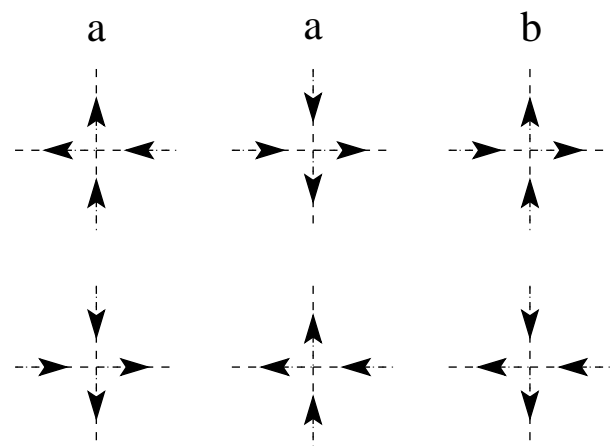

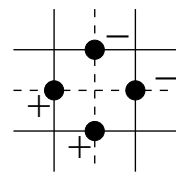

$\mathrm{b}$
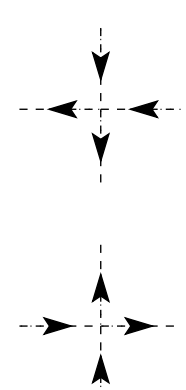

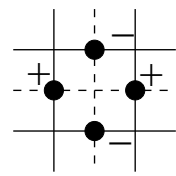

$\mathrm{c}$
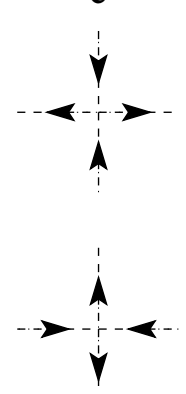
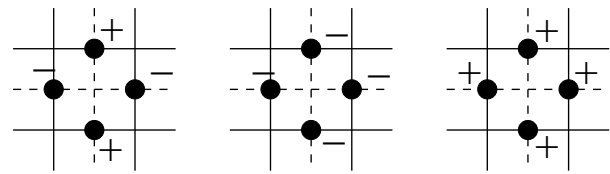

$\mathrm{c}$

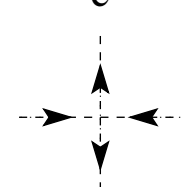

d
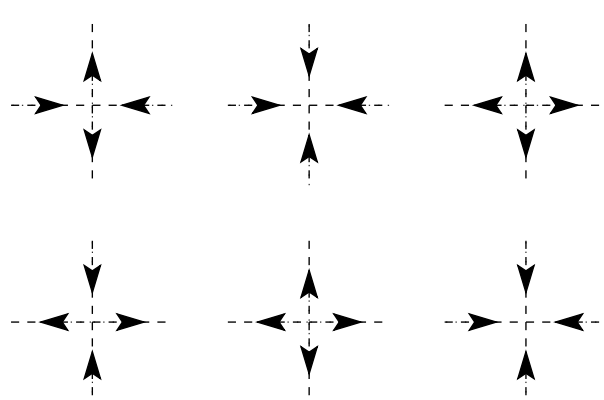

FIG. 5: Illustration of the eight allowed spin configurations around a plaquette of the square lattice, in any basis state $g|0\rangle, g \in G$. These can in turn be mapped onto the configurations of an eight vertex model by replacing each positive spin with an arrow along the corresponding bond of the dual lattice (dashed lines), pointing, say, from sublattice $A$ to sublattice $B$ (vice versa for the negative spins). Clearly, the corresponding eight vertex configurations differ depending on the location of the plaquette in the dual lattice: sublattice $A$ (middle), or sublattice $B$ (bottom). The letters $a, b, c, d$ correspond to the usual labeling of the vertex fugacities in the eight vertex model.

the $(2+1 \mathrm{D})$ quantum system to those of a simple $(2 \mathrm{D}$, not 3D) classical Ising model at an inverse (classical) temperature equal to the value of the coupling constant $\beta$ that drives the quantum system through the $T=0$ phase transition. For example, the magnetization of the quantum system equals the energy $E_{\text {Ising }}(\beta)$ of the classical Ising model. While the magnetization is continuous and non-vanishing across the quantum phase transition (much as the energy $E_{\text {Ising }}(\beta)$ is across the classical Ising transition), its derivative with respect to $\beta$ diverges logarithmically at $\beta_{c}$ (much as the Ising model heat capacity $C_{\text {Ising }}$ diverges logarithmically at $\beta_{c}$ ).

Despite the relation to the $2 \mathrm{D}$ classical Ising model, the quantum phase transition does not have a local order parameter that vanishes on one side and not on the other. Of course one expects that no local order parameter can characterize the topological phase, but in this particular example, there is no order parameter that characterizes the non-topologically ordered phase either. One can indeed identify from the mapping to the Ising model a parameter that orders in the nontopological phase; however, this variable is non-local in the physical spin variables used to define the local Hamiltonian. Specifically, the order parameter is, in the language used in this paper, the expectation value of the $\theta_{s}$ variables defined on the sites of the square lattice, such that $\sigma_{\left\langle s s^{\prime}\right\rangle}=\theta_{s} \theta_{s}^{\prime}$, for nearest neighboring sites $s, s^{\prime}$. While $\sigma_{\left\langle s s^{\prime}\right\rangle}$ is obviously local in terms of the $\theta_{s}, \theta_{s}^{\prime}$, the inversion needed to write the $\theta$ 's in terms of the $\sigma$ 's is non-local. Hence, $\left\langle\theta_{s}\right\rangle$ may detect the transition into the non-topological phase, but as it is non-local it is not an order parameter in the usual sense. That there is no order parameter for the non-topologically ordered phase is not generic (see the example in Sec. IIIB), as perhaps the most obvious exit from a topological phase is by escaping into a locally ordered phase due to spontaneous symmetry breaking. Hence, the main example studied in this paper is particularly interesting in that one has no local order parameter in either phases.

Recently, P. Zanardi et al. proposed a new approach to study quantum phase transitions through the behavior of a metric tensor in parameter space, derived from a fidelity-based notion of distance between states ${ }^{20}$ In this approach, one does not require any a priori knowledge of an order parameter to detect a phase transition (one may argue that the fidelity encompasses correlations of both local and non-local operators). Thus, these ideas may be particularly useful to detect topological quantum phase transitions (see Ref. 9).

We end with a speculative note: topological quantum phase transitions should share the feature that, even in the case when there is no local order parameter in either the topological or the non-topological phase, there should be, generically, detectable singularities in high enough derivatives of local observables with respect to the coupling that takes the system across the transition (as in the case study presented here). ${ }^{21}$ After all, what would be a phase transition without singularities in any physical observable?

Upon completion of this work, we became aware of similar work being pursued from a gauge theoretical perspective by S. Papanikolaou, K. Raman and E. Fradkin on the quantum eight-vertex model, ${ }^{22}$ to which we refer the reader for a complementary approach.

\section{Acknowledgments}

We would like to thank Xiao-Gang Wen, Eduardo Fradkin and Stefanos Papanikolaou for enlightening discussions. We are particularly indebted to Paul Fendley, whose comments brought us to consider the model discussed in Sec. IIIB This work is supported in part by the NSF Grants DMR-0305482 
and DMR-0403997 (CC and CC), and by EPSRC Grant No. GR/R83712/01 (C. Castelnovo). C. Castelnovo would like to acknowledge the I2CAM NSF Grant DMR No. 0645461 for travel support, during which part of this work was carried out.
1 X.-G. Wen, Int. J. Mod. Phys. B 4, 239 (1990); Adv. in Phys. 44, 405 (1995); Phys. Rev. B 65, 165113 (2002).

${ }^{2}$ F. D. M. Haldane, and E. H. Rezayi, Phys. Rev. B 31, 2529 (1985).

3 X.-G. Wen, and Q. Niu, Phys. Rev. B 41, 9377 (1990).

${ }^{4}$ D. Arovas, J. R. Schrieffer, and F. Wilczek, Phys. Rev. Lett. 53, 722 (1984).

5 A. Y. Kitaev, Ann. Phys. (N.Y.) 303, 2 (2003).

${ }^{6}$ M. Levin, and X.-G. Wen, Phys. Rev. B 75, 075116 (2007).

7 A. Hamma and D. A. Lidar, (accepted for publication on Phys. Rev. Lett.), arXiv:quant-ph/0607145 v4 (2006).

${ }^{8}$ S. Trebst, P. Werner, M. Troyer, K. Shtengel, and C. Nayak, Phys. Rev. Lett. 98, 070602 (2007).

9 A. Hamma, W. Zhang, S. Haas, and D. A. Lidar, arXiv:0705.0026v1 (2007).

10 M. Levin, and X.-G. Wen, Phys. Rev. Lett. 96, 110405 (2006).

11 A. Y. Kitaev, and J. Preskill, Phys. Rev. Lett. 96, 110404 (2006).

12 A. Hamma, R. Ionicioiu, and P. Zanardi, Phys. Rev. A 71, 022315 (2005).

13 C. Castelnovo, C. Chamon, C. Mudry, and P. Pujol, Ann. Phys. (N.Y.) 318, 316 (2005).

${ }^{14}$ E. Fradkin, and J. E. Moore, Phys. Rev. Lett. 97, 050404 (2006).

15 C. Castelnovo and C. Chamon, Phys. Rev. B 76, 174416 (2007).
16 A. Bugrij, and V. N. Shadura, JETP Lett. 63, 384 (1996); arXiv:hep-th/9601106

17 This condition is actually immaterial, and can be avoided using an approach similar to the one by Furukawa and Misguich [Phys. Rev. B 75, 214407 (2007)].

18 E. Ardonne, P. Fendley, and E. Fradkin, Ann. Phys. (N.Y.) 310 , 493 (2004).

19 R. Baxter, Exactly Solved Models in Statistical Mechanics (Academic Press, London, 1982).

${ }^{20}$ P. Zanardi, L. Campos Venuti, P. Giorda, Phys. Rev. A76, 062318 (2007), and references therein. The behavior of fidelity across a topological phase transition in the Kitaev model in presence of a magnetic field was studied numerically in Ref.9.

${ }^{21}$ A similar behavior - i.e., the lack of a local order parameter but display of local non-analyticities - was recently argued by Z. Nussinov and G. Ortiz to characterize as well a temperature-driven topological phase transition in the Kitaev model [arXiv:cond-mat/0702377].

22 S. Papanikolaou, K. Raman, and E. Fradkin, Phys. Rev. B 76, 224421 (2007). 